\title{
High Data Rate Optical logic OR, and NOT Gates at Optimum Injection Current based on SOA-MZI
}

\author{
Azhar Hussein Neama \\ Electrical Engineering Department \\ College of Engineering, University of Baghdad \\ Baghdad, Iraq
}

\author{
Ismael Shanan Desher \\ Electrical Engineering Department \\ College of Engineering, University of Baghdad \\ Baghdad, Iraq
}

\begin{abstract}
In this paper, the semiconductor optical amplifier (SOA) is used with a Mach-Zehnder interferometer (MZI) forming an SOA-MZI structure which is used to perform the logic gates OR, and NOT. It is simulated at $10 \mathrm{Gbps}, 20 \mathrm{Gbps}$, and 40 Gbps to extract simple design rules. Two binary input data signals are simulated at several bitrate $10 \mathrm{Gbps}, 20 \mathrm{Gbps}$, and 40 Gbps. It is demonstrated that high data rate can be achieved with a specific injection current in SOA. SOAs required low injection current, which leads to a low value on the total power consumption of the gate. In addition, this work includes the study of the effect of the bit rate on the received power, minimum bit error rate (BER), and maximum quality factor (Q-factor). The logical output of the gate has an extinction ratio of more than $10 \mathrm{~dB}$ with good eye opening. The output of the OR, NOT gates shows error-free operation at different bit rate with a clear eye opening.
\end{abstract}

\section{General Terms}

Optical amplifier, MZI, optical logic gates.

\section{Keywords}

SOA, XPM, FWM, XGM, injection current.

\section{INTRODUCTION}

In future high-speed telecommunication systems, all-optical signal processing techniques are distinguished to avoid optical electrical transformations that may create data-flow bottlenecks. Unitary NOT function is the simplest Boolean functions which performs a logical inversion of the input data. Logic OR gate is one of the logical gates that has not been demonstrated as wide as XOR and AND gates since the later gates have found more extensive applications for simple optical signal processing. Various approaches are proposed to achieve all optical gates. In the paper all Optical Gates based semiconductor optical amplifier (SOA) are used which are mainly based on SOA nonlinear phenomena like cross gain modulation (XGM), cross phase modulation (XPM), and four wave mixing (FWM) making SOAs attractive for all optical signal processing such as all optical switching and wavelength conversion. In addition, it can be integrated with semiconductors, lasers, modulators, etc. [1], [2]. Moreover, SOA have advantages in terms of low-power consumption, a small footprint, and homogeneous integration [3].

Thence SOAs have been widely used to perform a variety of all-optical functions such as wavelength conversion [4], signal regeneration [5], pulse reshaping [6], and power limiting [7]. Compared to their fiber-based counterparts [8]. Nevertheless such techniques also have one serious limitation. While the nonlinear response of optic fiber is nearly immediate[9], the nonlinear response time of a SOA is related to its carrier lifetime [10] that governs the speed of recovery of SOA gain typically exceeds 0.1 ns.. Many developments have been reported to implement different optical systems in different techniques. Each optical processing provide a smart solution to avoid time-consuming optical conversion in different networks.

Mach-Zehender Interferometer (MZI), as each arm incorporates an SOA is used to transform phase modulates to intensity modulation Thus, owing to the phase change in between two signals propagating through the arms constructive and destructive interference occur. Also, by operating on the negative slope of the interferometer transfer function logical inversion can be obtained.

One of the simplest methods to speed up the gain recovery in SOAs is increasing the applied bias current. A high current provides a large carrier density and also a high amplified spontaneous emission power, both of which tend to shorten the carrier lifetime. Therefore, to obtain a fast gain recovery, a high current must be applied. In this work an optimum injection current is calculated for each data rate.

\section{SOA PARAMETERS SPECIFICATION}

Changes in terms of the gain occur within the active region of the SOA by injecting light and bias current into the SOA. Using a gain characteristics equation is to describe these changes. The unsaturated gain coefficient, $g_{0}$ describe the unsaturated gain medium of the amplifier by which is given by [11]:

$$
g_{0}=\Gamma \mathrm{a}\left(\frac{I \tau_{c}}{\mathrm{eV}}-n_{0}\right)
$$

Where $\Gamma$ is the confinement factor, a is the material gain, $I$ is the injected current into the SOA, $\tau_{c}$ is the carrier lifetime, $e$ is the charge of the electron and $n_{0}$ is the carrier density at transparency point. $\mathrm{V}$ is the active volume of the SOA. The single pass gain of SOA, which is also known as the unsaturated gain, $G_{0}$ of the amplifier, is given by:

$$
G_{0}=\exp \left(g_{0 L}\right)
$$

From eq. 1 can be transformed into this form:

$$
I=\frac{e V}{\tau_{c}}\left(\frac{g_{0}}{\Gamma a}+n_{0}\right)
$$

From eq. 3 it can be observed that the injection current I directly proportional $1 / \tau_{c}$ in other word I $\alpha$ bitrate, here the repetition rate is used much greater than $1 / \tau_{c}$, there are negligible signal distortion or pattern effects.

The SOA parameters used in the simulation are clarify as in Table1. 


\begin{tabular}{|c|c|c|c|}
\hline P.NO. & $\begin{array}{c}\text { Table } 1 \text { Physical SO } \\
\text { Name }\end{array}$ & $\begin{array}{l}\text { parameters } \\
\text { Value }\end{array}$ & Unit \\
\hline 1 & Length & 0.0005 & $\mathrm{~m}$ \\
\hline 2 & Width & $3 e-006$ & $\mathrm{~m}$ \\
\hline 3 & Height & $8 \mathrm{e}-008$ & $\mathrm{~m}$ \\
\hline 4 & $\begin{array}{l}\text { Optical confinement } \\
\text { factor }\end{array}$ & 0.3 & \\
\hline 5 & Loss & 0 & $1 / \mathrm{m}$ \\
\hline 6 & Differential gain & $2.78 \mathrm{e}-020$ & $\mathrm{~m}^{\wedge} 2$ \\
\hline 7 & $\begin{array}{l}\text { Carrier density at } \\
\text { transparency }\end{array}$ & $1.4 \mathrm{e}+024$ & $\mathrm{~m}^{\wedge} 3$ \\
\hline 8 & $\begin{array}{c}\text { Line width } \\
\text { enhancement factor }\end{array}$ & 5 & \\
\hline 9 & $\begin{array}{c}\text { Recombination } \\
\text { coefficient A }\end{array}$ & $\begin{array}{c}14300000 \\
0\end{array}$ & $1 / \mathrm{s}$ \\
\hline 10 & $\begin{array}{l}\text { Recombination } \\
\text { coefficient B }\end{array}$ & 1e-016 & $\mathrm{m}^{\wedge} 3 / \mathrm{s}$ \\
\hline 11 & $\begin{array}{l}\text { Recombination } \\
\text { coefficient } \mathrm{C}\end{array}$ & $3 e-041$ & $\mathrm{~m}^{\wedge} 6 / \mathrm{s}$ \\
\hline 12 & Initial carrier density & $3 e+024$ & $m^{\wedge}-3$ \\
\hline
\end{tabular}

\section{DESIGN AND SIMULATION}

\subsection{Optical OR gate at high bit}

This Boolean operation gives a logic " 1 " each time one (at least) of the input data bits is "1". So the output of this operation only will become a " 0 " in the case $A=0$ and $B=0$. This scheme is based also in the use of a SOA-MZI.

\subsection{Optical NOT gate at high bit}

This operation of one input port and one output port inverts the input signal, so whenever there is a logic " 1 " at the input, a logic " 0 " is obtained at the output. The NOT logic gate here presented is based in the SOA-MZI configuration.

\section{PRINCIPLE OF OPERATION}

\subsection{Principle of operation of optical OR gate}

Here, the concept of cross gain modulation (XGM) is used to perform the OR operation. The simplest case based on SOAMZI is that in which no wavelength conversion is used. In this case, the principle of operation, which is depicted in Figure 1. The two data signals are coupled at the same port, in this case port \#1. After passing through one of the two SOAs (in this case, the upper one), the amplified signal is coupled out at port \#4, carrying the OR operation between the two inputs. In this case, the two inputs must be at the same wavelength and the output signal is at the same wavelength than the input signals. There is no need to use a $\mathrm{CW}$ for performing this operation. In this case, the two inputs must beat the same wavelength and the output signal is at the same wavelength like the input signals. No wavelength conversion is done because is not needed.

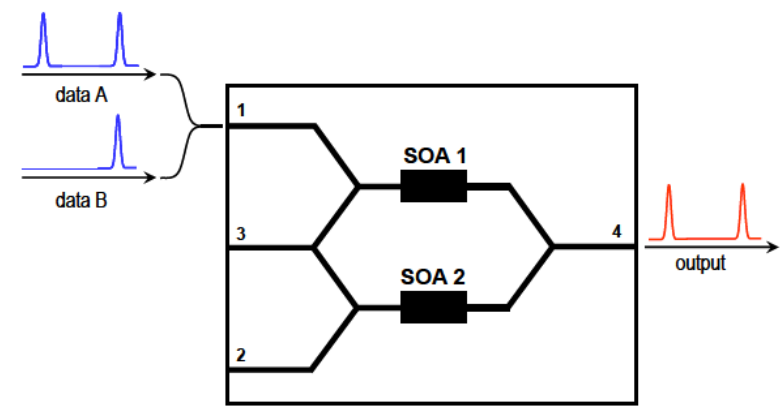

Fig 1: Principle of operation of the OR logic gate using a SOA-MZI without wavelength conversion.

The output is obtained at the same wavelength at which the data signal is generated. The two data signals are generated at $1556 \mathrm{~nm}$ and $0.3 \mathrm{~mW}$ power.

\subsection{Principle of operation of optical NOT gate}

The principle of operation of the NOT logic functionality based in SOA-MZI is depicted in Figure 2. There are two signals involved in this process: the original input data, and a train of optical pulses. The input data is the one to be inverted. The train of optical pulses is used to: a) generate the control signal, and b) generate an auxiliary data sequence. The input data sequence enters the SOA-MZI in port \#1. The auxiliary signal, which consists in a train of pulses, is coupled into port \#3 of the SOA-MZI, meanwhile the control signal is launched into port \#4. The output is obtained at port \#3.

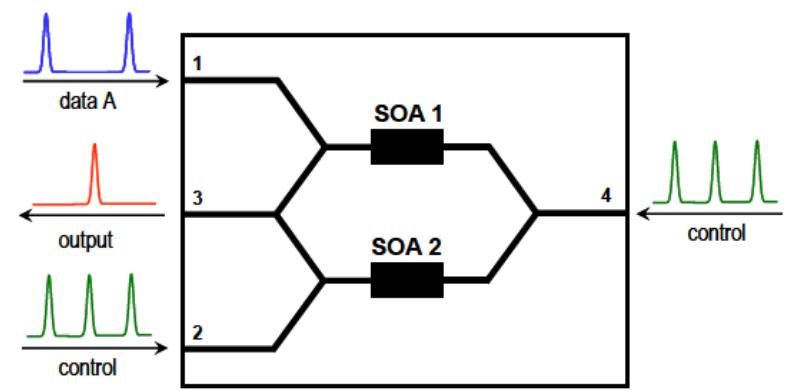

Fig 2: Principle of operation of the NOT logic gate using a SOA-MZI.

The control signal enables the operation of the gate. These pulses are split and travel through the upper and lower SOAs, following symmetric paths. The lower SOA conditions will be affected by the optical pulses that are entering into port \#2 (there is always an optical pulse at this port). If no pulse is present at port $\# 1$, i.e. $A=0$, the control pulse counterpropagating through both SOAs will experience different conditions, and the initial $\pi$ phase shift that the two versions of the control pulse would have at port \#3 is no longer held, and non-destructive interference is obtained. This means that a logic "1" will appear at the output. On the other hand, if $A=1$ the SOA conditions are the same so no differential phase shift is induced between the two version of the control pulse, and these will interfere destructively (due to the $\pi$ phase shift induced by the $3-\mathrm{dB}$ couplers at the input and output ports), resulting in no optical signal at the output (logic "0"). As a result, an inverted version of the original input data sequence is obtained at the output. 


\section{SIMULATION STEPS}

The parameters used in simulation in this paper is shown in Table 2

Table 2 Description of Parameters used in simulation.

\begin{tabular}{cccc}
\hline $\begin{array}{c}\text { P.N } \\
\text { O. }\end{array}$ & Description & Value & Unit \\
\hline $\mathbf{1}$ & $\begin{array}{c}\text { the data signal power at } 10 \\
\text { Gbps,20 Gbps and 40 Gbps }\end{array}$ & 0.3 & $\mathrm{~mm}$ \\
$\mathbf{2}$ & the CW signal power & 0.25 & $\mathrm{~mm}$ \\
$\mathbf{3}$ & the unsaturated gain, $G_{0}$ & 29 & $\mathrm{~dB}$ \\
$\mathbf{4}$ & pulse width at $10 \mathrm{Gbps}$ & 0.05 & $\mathrm{~ns}$ \\
& at $20 \mathrm{Gbps}$ & 0.025 & $\mathrm{~ns}$ \\
\hline $\mathbf{5}$ & at $40 \mathrm{Gbps}$ & 0.0125 & $\mathrm{~ns}$ \\
\hline & Carrier lifetime, $\boldsymbol{\tau}_{c}$ at $10 \mathrm{Gbps}$ & 1 & $\mathrm{~ns}$ \\
\hline & at $20 \mathrm{Gbps}$ & 0.5 & $\mathrm{~ns}$ \\
$\mathbf{6}$ & at $40 \mathrm{Gbps}$ & 0.25 & $\mathrm{~ns}$ \\
\hline & Injection current at $10 \mathrm{Gbps}$ & 57.7 & $\mathrm{~mA}$ \\
\hline & at $20 \mathrm{Gbps}$ & 115.4 & $\mathrm{~mA}$ \\
\hline & at $40 \mathrm{Gbps}$ & 230 & $\mathrm{~mA}$ \\
\hline
\end{tabular}

\subsection{Simulation Steps of optical OR gate}

The simulation setup of SOA-MZI based OR gate using Optisystem 7.0 software is shown in Figure 3.

The architectures is based on SOA-MZI. This is a simplest case because no wavelength conversion is used. The two data signals are coupled at the same port. The OR operation between the two inputs is carrying after passing through one of the two SOAs (in this case, the upper one).

\subsection{Simulation Steps of optical NOT gate}

Optiwave Photonic Design automation software Optisystem 7.0 is also used for performing simulation process.

The input data is the one to be inverted. The input data sequence enters the SOA-MZI in port \#1. The control signal enters in to port\#2 of the SOA-MZI. At the output the NOT gate operation is obtained.

The simulation setup of SOA based NOT gate is shown in Figure

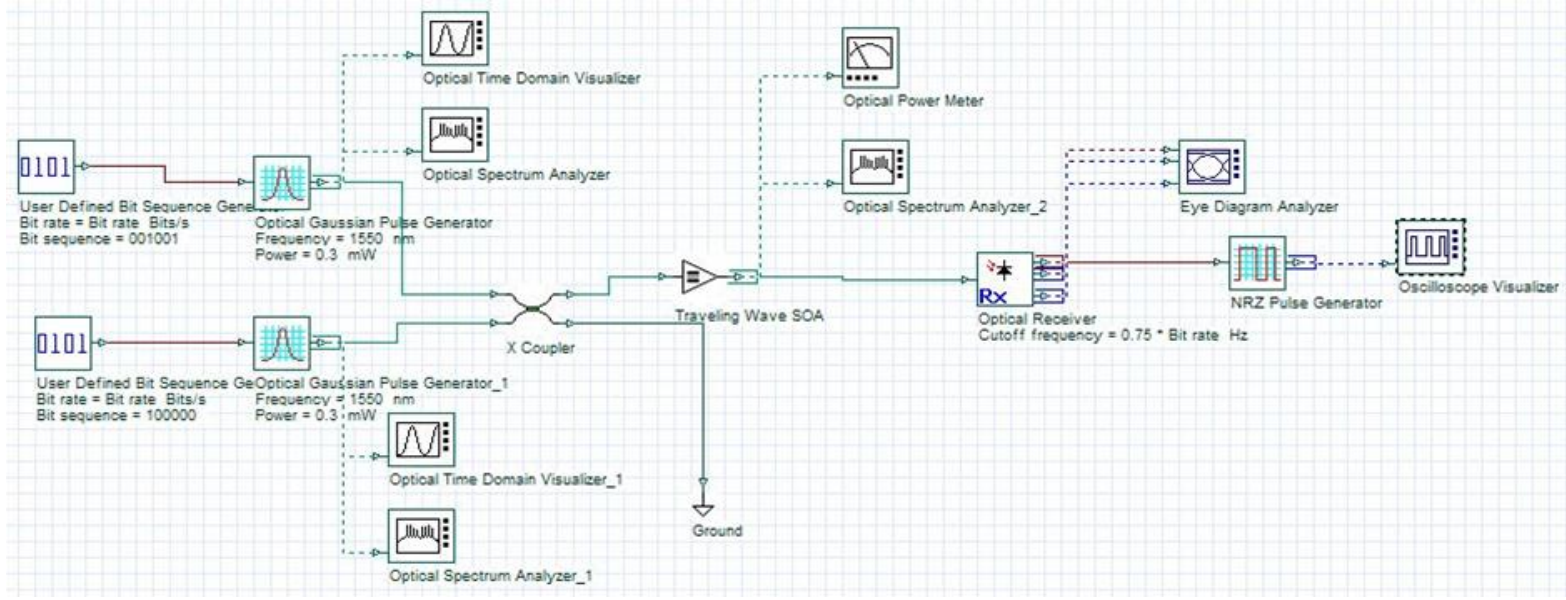

Fig 3: Simulation set up of SOA based OR Gate

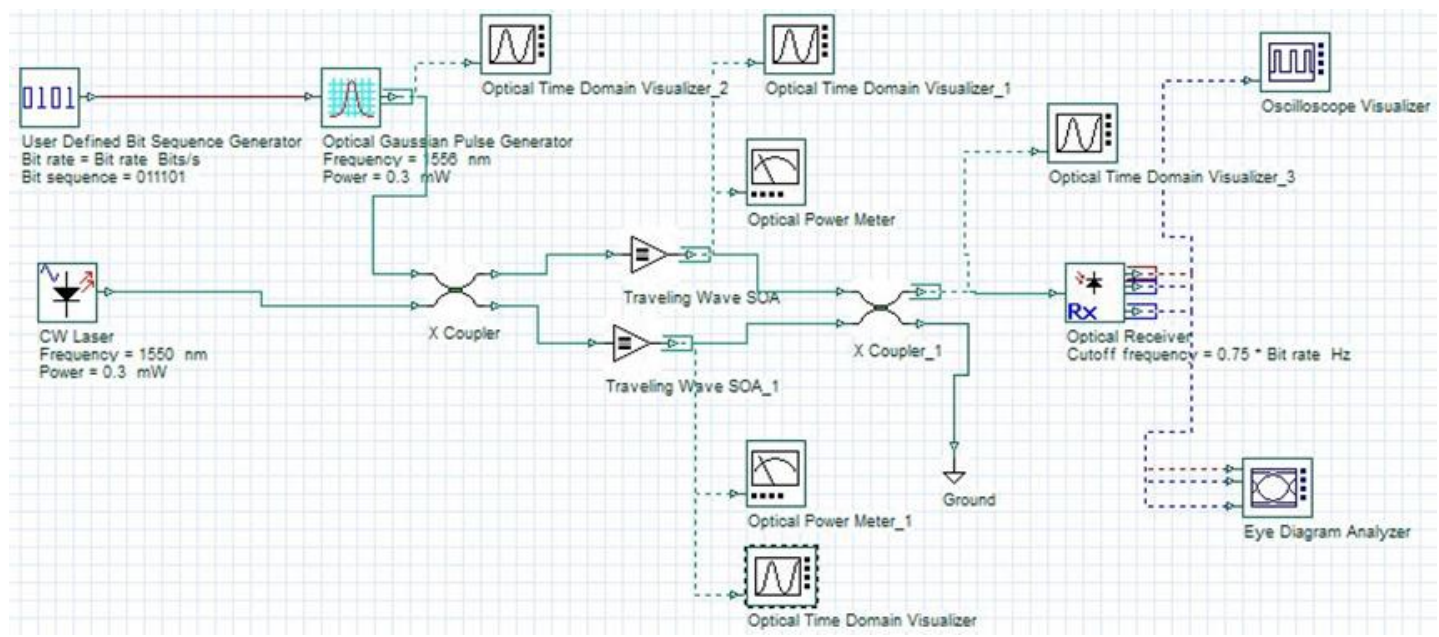

Fig 4: simulation of NOT gate

\section{RESULTS}

\subsection{Results of optical OR gate}

A lower value of output power will be obtained when FWM will be generated and output will be considered as logic " 0 ".
For output power calculation time domain visualizer and power meter has been used for different input sequences.

Table 3 summarizes and compares the simulation results of OR at 10, 20, and 40 Gbps bitrate optical logic gates. For 
system performance evaluation, Table 3 includes the output peak power, Q-factor, BER, and total received power.

Table 3 Summary of simulation results of OR optical logic gates at 10, 20, and 40Gbps bitrate

\begin{tabular}{|c|c|c|c|c|c|c|}
\hline S.NO. & $\mathbf{A}$ & B & $\begin{array}{c}\text { Output } \\
\text { power } \\
\text { (w) at } \\
10 \text { Gbps }\end{array}$ & $\begin{array}{c}\text { Output } \\
\text { power } \\
\text { (w) at } \\
20 \text { Gbps }\end{array}$ & $\begin{array}{c}\text { Output } \\
\text { power }(w) \text { at } \\
40 \mathrm{Gbps}\end{array}$ & $\begin{array}{l}\text { Logi } \\
\text { c }\end{array}$ \\
\hline 1 & 0 & 0 & $\begin{array}{c}586.094 \\
\text { E-12 }\end{array}$ & $81.883 \mathrm{E}-9$ & 41.906 E-6 & 0 \\
\hline 2 & 0 & 1 & $\begin{array}{c}19.584 \\
\text { E-3 }\end{array}$ & $\begin{array}{c}58.725 \text { E- } \\
3\end{array}$ & $142.805 \mathrm{E}-3$ & 1 \\
\hline 3 & 1 & 0 & $\begin{array}{c}19.584 \\
\text { E-3 }\end{array}$ & $\begin{array}{c}58.725 \mathrm{E}- \\
3\end{array}$ & 142.805 E-3 & 1 \\
\hline 4 & 1 & 1 & $\begin{array}{c}22.396 \\
\text { E-3 }\end{array}$ & $\begin{array}{c}62.476 \mathrm{E}- \\
3\end{array}$ & 147.485 E-3 & 1 \\
\hline $\begin{array}{l}\text { Max. Q } \\
\text { factor }\end{array}$ & & & 5.482 & 4.65 & 4. 41 & \\
\hline $\begin{array}{l}\text { Min. } \\
\text { BER }\end{array}$ & & & $1.2 \mathrm{e}-008$ & $9.77 \mathrm{e}-007$ & $3.101 \mathrm{e}-006$ & \\
\hline
\end{tabular}

Figure 5 displays the eye pattern for an all-optical OR gate at different bitrate. It is clear that, the performance of the gate at $10 \mathrm{Gbps}$ bitrate is the best one, where the eye opening is maximum. It is worth mentioning that due to the consecutive ones in the chosen code, the BER of the 40 Gbps decreases.

In this setup only two data signals are used (Figures 6(a) (b) at $10 \mathrm{Gbps}(\mathrm{d})(\mathrm{e})$ at $20 \mathrm{Gbps}$ and (g) (h) at $40 \mathrm{Gbps}$ ) which are used to perform OR operation. There is no need to use a CW for performing this operation. In this case, the two inputs must be at the same wavelength and the output signal is at the same wavelength than the input signals. No wavelength conversion is done. The output is obtained at the same wavelength at which the data signal is generated. These two signals are coupled at the two ports of coupler and given to SOA (Figure 6(c) at $10 \mathrm{Gbps} 6(\mathrm{f})$ at $20 \mathrm{Gbps} 6(\mathrm{i})$ at $40 \mathrm{Gbps}$.

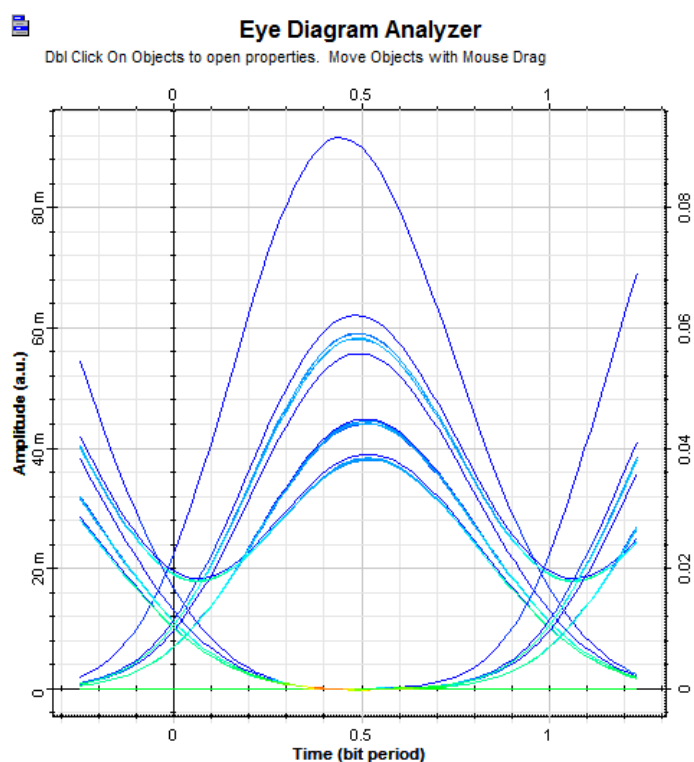

(a)

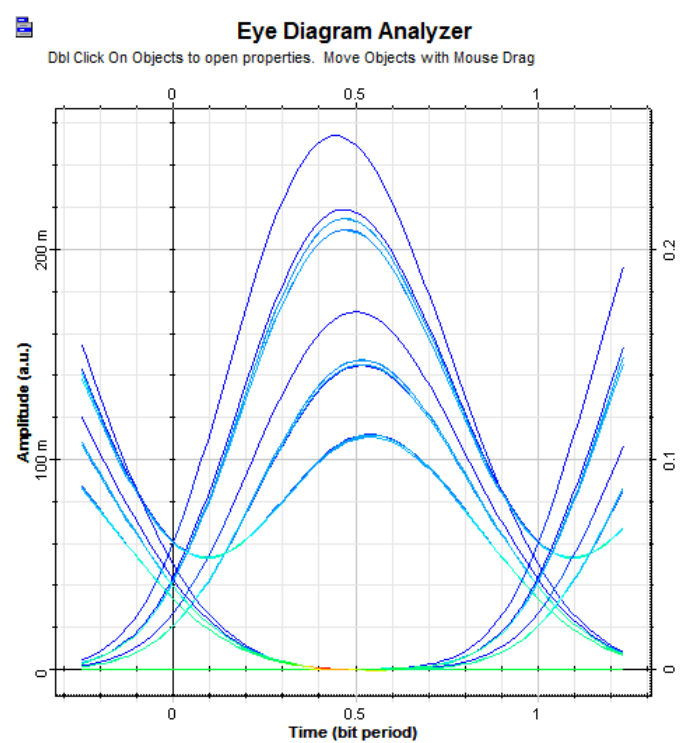

(b)

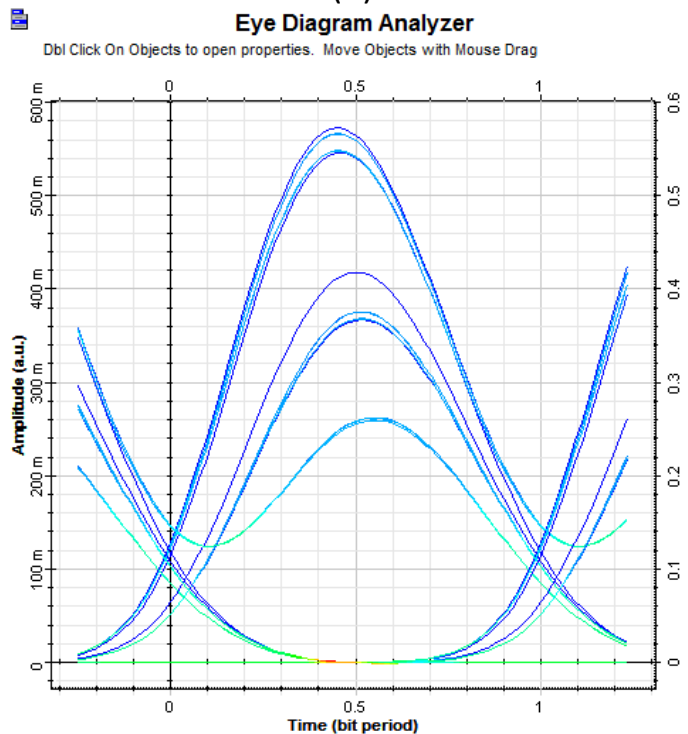

(c)

Fig 5: Eye diagram simulation results for OR gate at (a) 10 Gbps, (b) 20 Gbps, and (c) 40 Gbps.

를 Optical Time Domain Visualizer Hold Control Key for Accelerated Panning

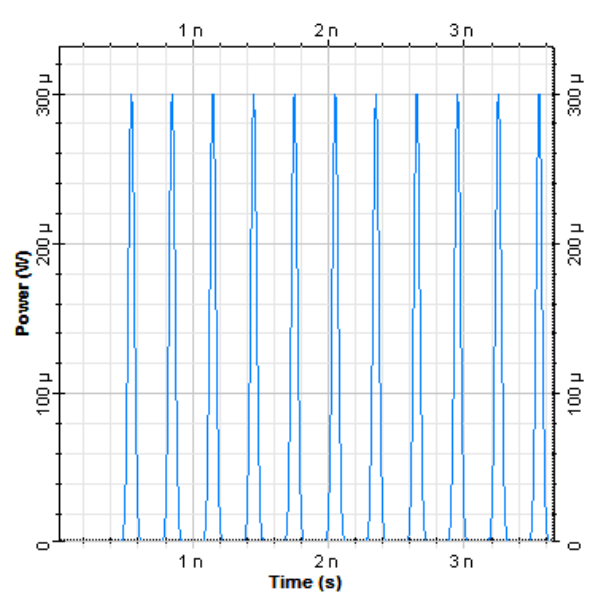

(a) 
宣

Optical Time Domain Visualizer_1 Hold Control Key for Accelerated Panning

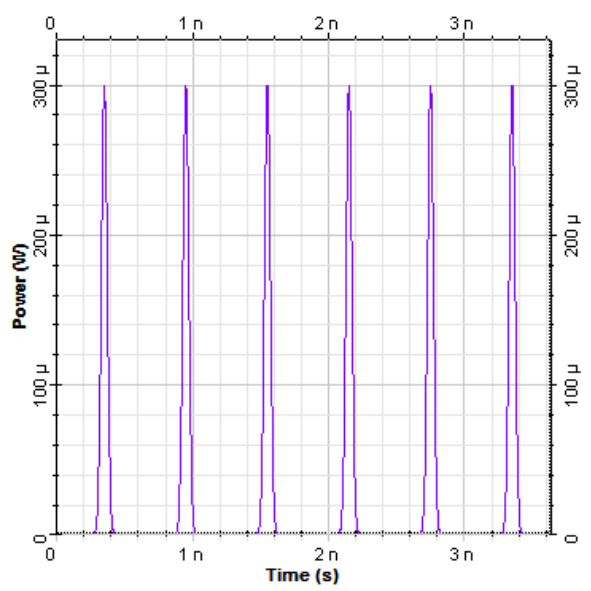

(b)

珰 Oscilloscope Visualizer Hold Control Key for Accelerated Panning

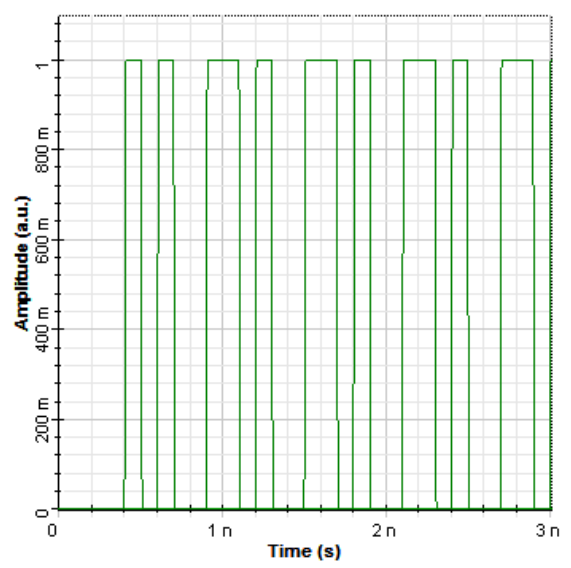

(c)
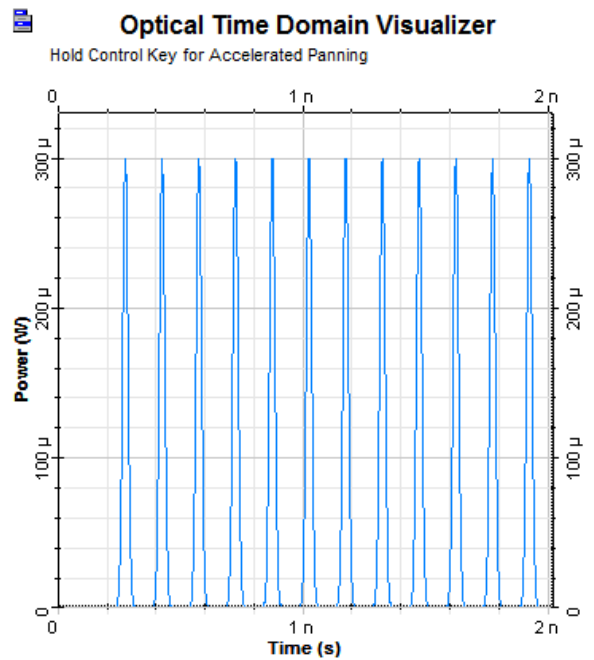

(d)
불 Optical Time Domain Visualizer_1 Hold Control Key for Accelerated Panning

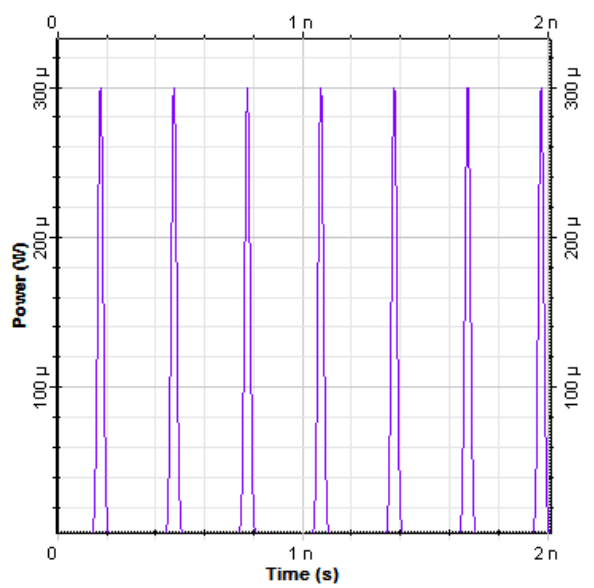

(e)
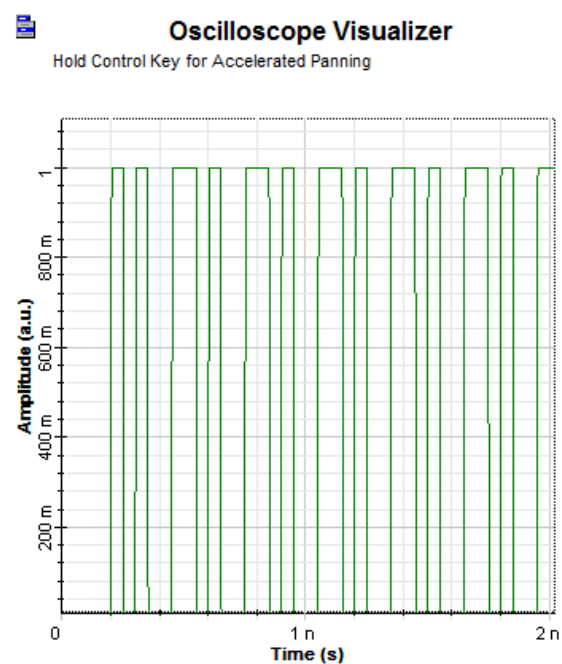

(f)
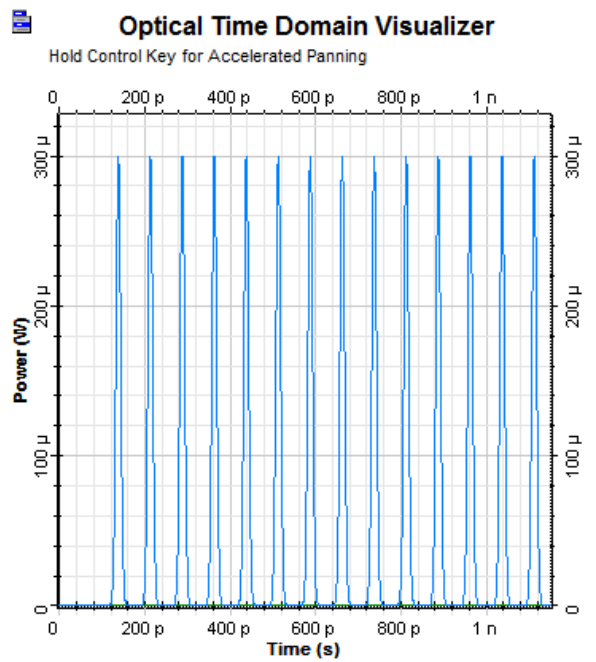

(g) 
圆

Optical Time Domain Visualizer_1

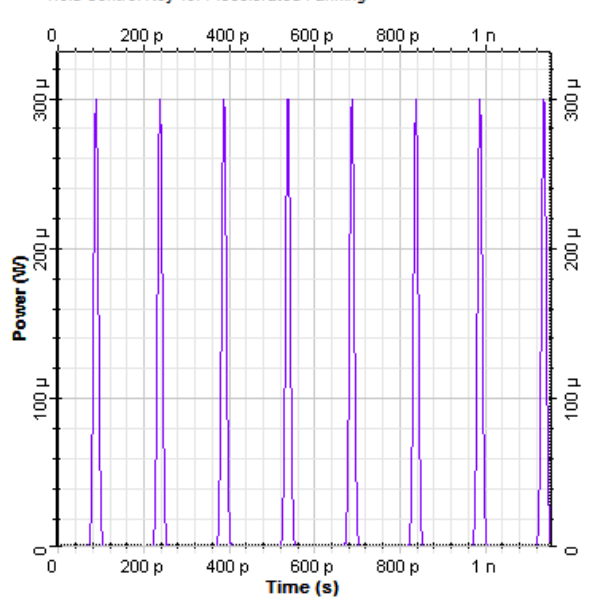

(h)

圆

Oscilloscope Visualizer
Left Button and Drag to Select Zoom Region. Press Control Key and Left

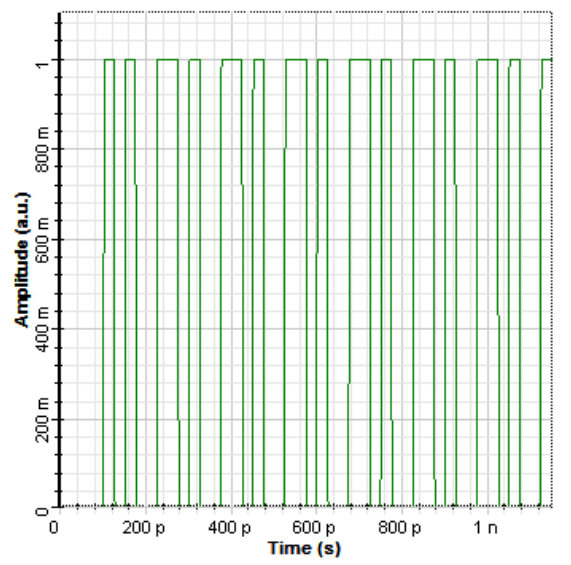

(i)

Fig 6: OR (a First Input Data (001001); (b) Second Data Input (100000); (c) optical output waveform showing signal peak power at 10 Gbps; (d First Input Data (001001); (e) Second Data Input (100000) (f) optical outputwaveform showing signal peak power at 20 Gbps; and (g First Input Data (001001); (h) Second Data Input (100000) (i) optical output waveform showing signal peak power at 40 Gbps.

Figure 7 shows the simulated result the results are observed in oscilloscope visualizer it can be observed that by simulate a SOA-BASED optical OR gate the logical expression are obtained as

$$
\text { Output }=\mathrm{A}+\mathrm{B}
$$

\subsection{Results of optical NOT gate}

There are two signals involved in this process: the original input data, which is the one to be inverted and the control signal. These signals injected into the amplifier, one is the signal carrying data at wavelength $1556 \mathrm{~nm}$ and the second a $\mathrm{CW}$ probe signal at wavelength $1550 \mathrm{~nm}$. When the data is in high state (1), the gain of the amplifier is saturated which reduces the gain on the probe at wavelength $1550 \mathrm{~nm}$ and hence the output at $1550 \mathrm{~nm}$ is reduced. When the data signal is low (0), the gain of the amplifier is not reduced and hence the gain on the probe at wavelength $1550 \mathrm{~nm}$ is high and hence the output at $1550 \mathrm{~nm}$ is high.

Thus cross gain modulation (XGM) leads naturally to an Invert operation. The process is also accompanied by cross phase modulation (XPM), i.e. the signal carrying data also modulates the phase of the co-propagating $\mathrm{CW}$ probe signal.

Table 4 summarizes and compares the simulation results of NOT at 10, 20, and 40 Gbps bitrate optical logic gates. For system performance evaluation, Table 3 includes the output peak power, Q-factor, BER, and total received power.

Table 4 Summary of simulation results of NOT optical logic gates at 10, 20, and 40Gbps bitrate

\begin{tabular}{|c|c|c|c|c|c|}
\hline S.NO. & $\mathbf{A}$ & $\begin{array}{c}\text { Output } \\
\text { power (w) } \\
\text { at } \\
10 \mathrm{Gbps}\end{array}$ & $\begin{array}{c}\text { Output } \\
\text { power } \\
\text { (w) at } \\
20 \\
\text { Gbps }\end{array}$ & $\begin{array}{c}\text { Output } \\
\text { power } \\
\text { (w) at } \\
\mathbf{4 0} \text { Gbps }\end{array}$ & Logic \\
\hline 1 & 0 & 74.172 E-3 & $\begin{array}{c}160.305 \\
\text { E-3 }\end{array}$ & $\begin{array}{c}337.344 \\
\text { E-3 }\end{array}$ & 1 \\
\hline 2 & 1 & $47.229 \mathrm{E}-3$ & $\begin{array}{c}96.656 \\
\text { E-3 }\end{array}$ & $\begin{array}{c}187.480 \\
\text { E-3 }\end{array}$ & 0 \\
\hline $\begin{array}{c}\text { Max. } \\
\mathbf{Q} \\
\text { factor }\end{array}$ & & 41.0243 & 28.235 & 34.2435 & \\
\hline $\begin{array}{l}\text { Min. } \\
\text { BER }\end{array}$ & & 0 & $\begin{array}{c}1.02 \mathrm{e}- \\
175\end{array}$ & $\begin{array}{c}2.38 \mathrm{e}- \\
257\end{array}$ & \\
\hline
\end{tabular}

For output power calculation time domain visualizer and power meter has been used for different input sequences.

The best place for determining whether a given bit is a " 1 " or a " 0 " is the sampling phase with the largest "eye opening."

The larger the eye opening, the greater the difference between the mean values of the signal levels for a " 1 " and a " 0 ." The greater that difference is, the higher the Q-factor and the better the BER performance. Note $Q$ value decreases with increasing gain recovery time.

Figure 9 displays the eye pattern for an all-optical NOT gate at different bitrate. It is clear that, the performance of the gate at $10 \mathrm{Gbps}$ bitrate is the best one, where the eye opening is maximum.

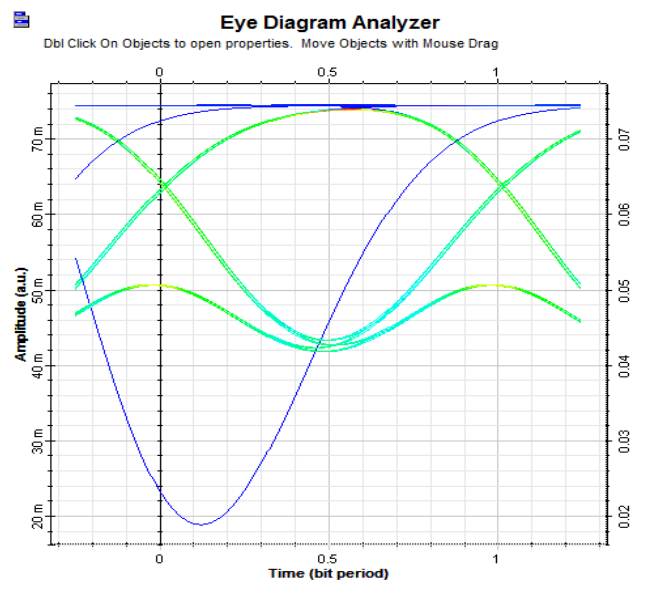

(a) 
园

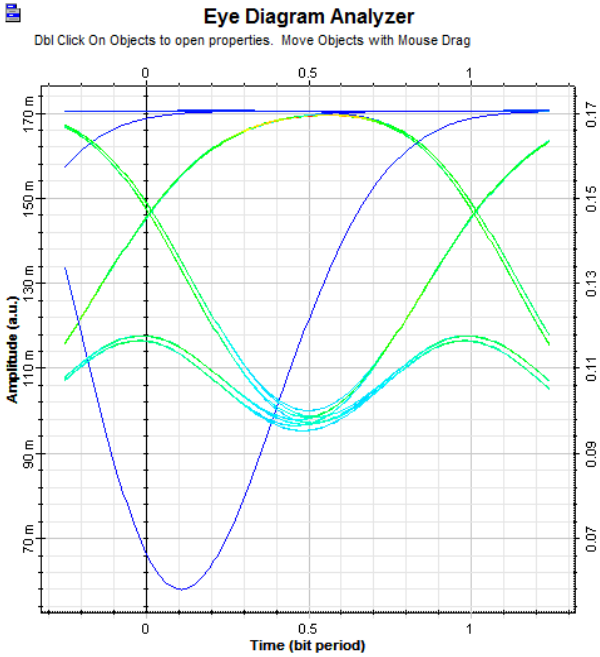

(b)

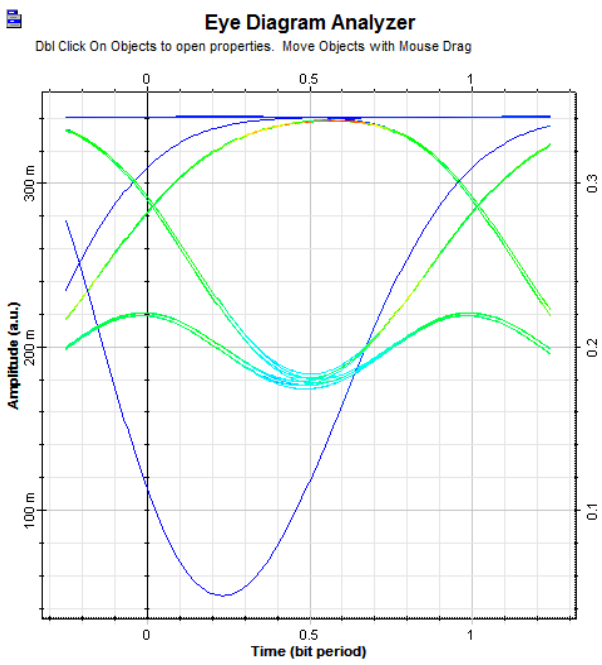

(c)

Fig 8: Eye diagram simulation results for NOT gate at (a) 10 Gbps, (b) 20 Gbps, and (c) 40 Gbps.

In this setup only two data signals are used (Figures 9(a) (b) at $10 \mathrm{Gbps}(\mathrm{d})$ (e) at $20 \mathrm{Gbps}$ and (g) (h) at $40 \mathrm{Gbps}$ ) which are used to perform NOT operation. The output is obtained at the same wavelength at which the data signal is generated. These two signals are coupled at the two ports of coupler and given to SOA (Figure 9(c) at $10 \mathrm{Gbps} 9$ (f) at $20 \mathrm{Gbps} 9$ (i) at 40 Gbps.
룰

Optical Time Domain Visualizer_2 Hold Control Key for Accelerated Panning

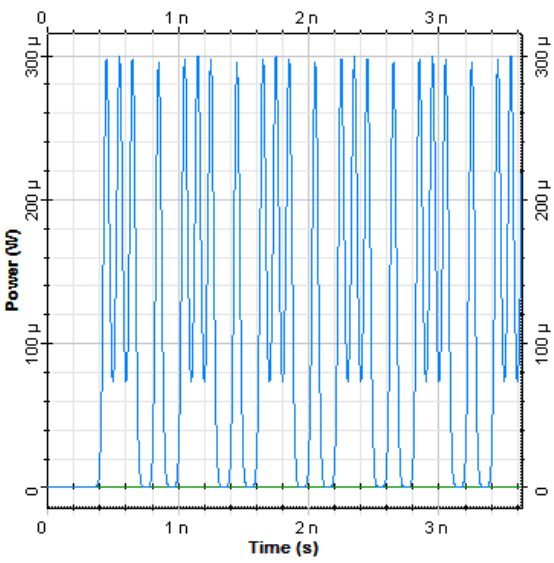

(a)
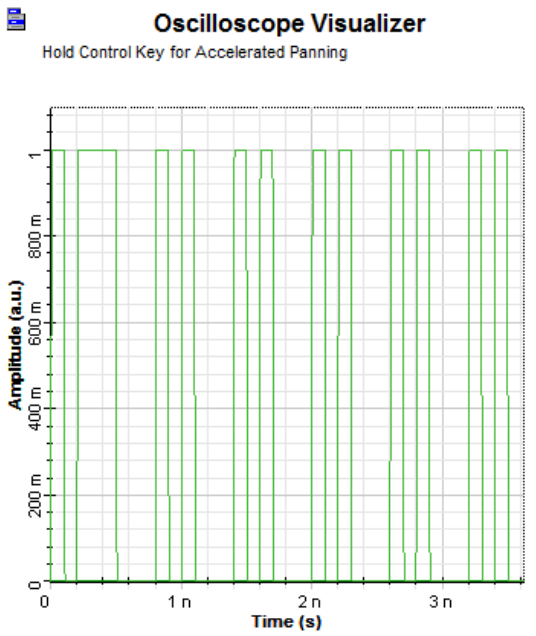

(b)

를 Optical Time Domain Visualizer_2 Hold Control Key for Accelerated Panning

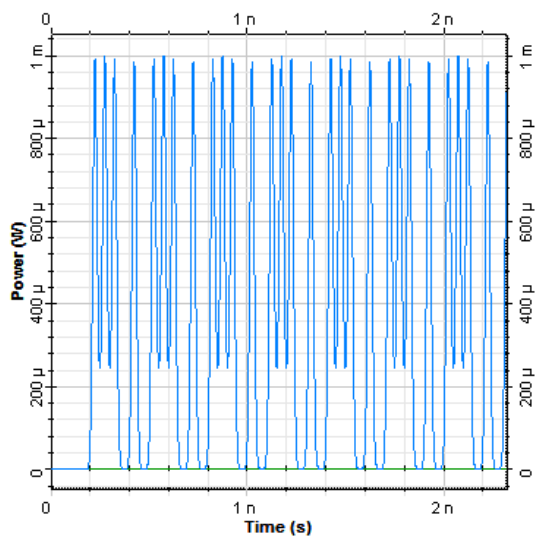

(c) 
룹

Oscilloscope Visualizer
Dbl click On Objects to open properties. Move Objects with Mouse Drag

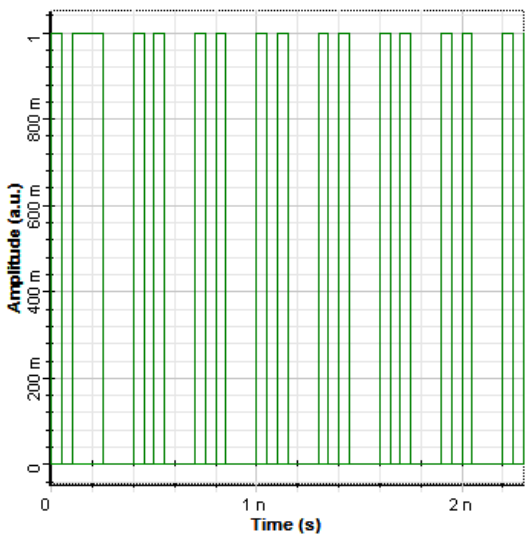

(d)
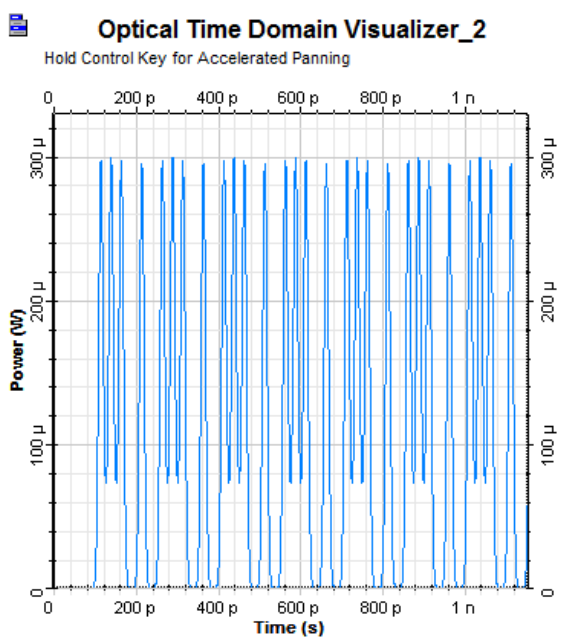

(e)

를
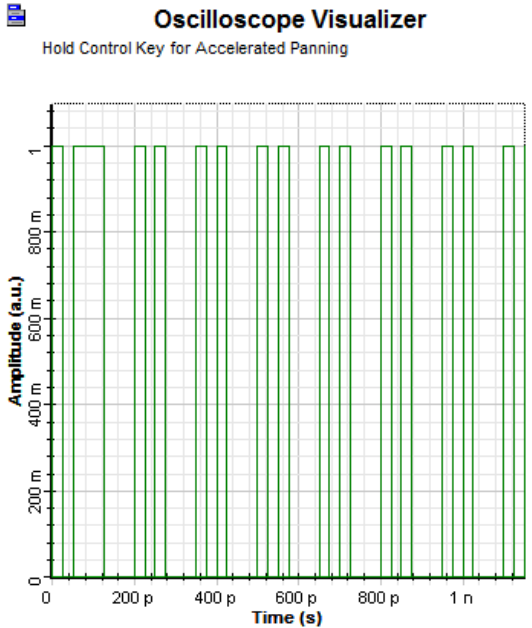

(f)

Fig 9: NOT (a Input Data (011101); (b) optical output waveform showing signal peak power at $10 \mathrm{Gbps}$; (c Input Data (011101); (d) optical output waveform showing signal peak power at 20 Gbps; (e Input Data (011101) (f) optical output waveform showing signal peak power at 40 Gbps.
Figure 9 shows the simulated result the results are observed in oscilloscope visualizer it can be observed that by simulate a SOA-BASED optical NOT gate which is represented by the logical expression as

$$
\text { Output }=\overline{\mathrm{A}}
$$

\section{CONCLUSION}

This paper simulates and demonstrates optical logic gates OR, and NOT using almost the same setup with some slight modifications inclusion of some components. The Boolean functionalities have been successfully demonstrated using low energy levels and without any additional pump signal. The parameters of SOA are optimized while simulating to get the output. SOAs required low injection current which leads to a low value on the total power consumption of the gate. The SOA-MZI turns out as a very promising candidate to implement all-optical logic gates because of its advantages. The quality factor ( $\mathrm{Q}$ factor) and Bit error rate (BER) have been analyzed for $10 \mathrm{Gbps}, 20 \mathrm{Gbps}$, and $40 \mathrm{Gbps}$.

\section{REFERENCES}

[1] M. J. Connelly, "Semiconductor Optical Amplifiers", Kluwer Academic Press, Boston, 2002.

[2] A. Rostami, H. Baghban, R. Maram, "Nanostructure Semiconductor Optical Amplifiers," Berlin, Germany: Springer-Verlag, 2011.

[3] T. Houbavlis, K. E. Zoiros, M. Kalyvas, G. Theophilopoulos, C. Bintjas, K. Yiannopoulos, N. Pleros, K. Vlachos, H. Avramopoulos, L. Schares, L. Occhi, G. Guekos, J. R. Taylor, S. Hansmann, and W. Miller, "All optical signal processing and applications within the Esprit project DO-ALL," IEEE J. Lightwave. Technol., vol. 23, no. 2, pp. 781-801, Feb. 2005

[4] J. Leuthold, R. Ryf, D. N. Maywar, S. Cabot, J. Jacques, and S. S. Patel, "Nonblocking all-optical cross connect based on regenerative all-optical wavelength converter in a transparent demonstration over 42 nodes and 16,800 km," J. Lightwave. Technol., vol. 21, no. 1, pp. 2863 2870, Nov. 2003.

[5] M. P. Dlubek, A. J. Phillips, and E. C. Larkins, "Extinction ratio improvement using nonlinear fourwave mixing in SOAs with assist beam," Microwave Opt. Technol. Lett., vol. 50, no. 8, pp. 2079-2083, Aug. 2008.

[6] G. Contestabile, M. Presi, and R. Proiletti, and E. Ciaramella, "Optical reshaping of 40-Gb/s NRZ and RZ signals without wavelength conversion," IEEE Photon. Technol. Lett., vol. 20, no. 15, pp. 1133-1135, July 2008.

[7] G. Contestabile, M. Presi, R. Proiletti, N. Calabretta, and E. Ciaramella, "A simple and low-power optical limiter for multi-GHz pulse trains," Opt. Express, vol. 15, no. 15, pp. 9849-9858, July 2007.

[8] P. V. Mamyshev, "All-optical regeneration based on selfphase modulation effect," in Proc. Eur. Conf. Optical Communication (ECOC98), Madrid, Spain, Sep. 20-24, 1998, pp. 475-476.

[9] G. P. Agrawal, "Nonlinear Fiber Optics", 4th ed., Academic Press, Boston, 2007.

[10] G. P. Agrawal and N. A. Olsson, "Self phase modulation and spectral broadening of optical pulses in 
International Journal of Computer Applications (0975 - 8887)

Volume 177 - No.3, November 2017

semiconductor laser amplifiers," IEEE J. Quantum Electron., vol. 25, no. 11, pp. 2297-2306, Nov. 1989.
[11] N. K. Dutta \& Q. Wang "semiconductor optical amplifier, "World Scientific Publishing Co. Pte. Ltd., New York, NY, USA, pp. 133, 2006. 